Tohoku Math. J.

68 (2016), 621-638

\title{
WINTGEN IDEAL SUBMANIFOLDS OF CODIMENSION TWO, COMPLEX CURVES, AND MÖBIUS GEOMETRY
}

\author{
Tongzhu Li, Xiang MA*, Changping Wang and ZhenXiao Xie
}

(Received December 4, 2014, revised April 2, 2015)

\begin{abstract}
Wintgen ideal submanifolds in space forms are those ones attaining the equality pointwise in the so-called DDVV inequality which relates the scalar curvature, the mean curvature and the scalar normal curvature. Using the framework of Möbius geometry, we show that in the codimension two case, the mean curvature spheres of the Wintgen ideal submanifold correspond to a 1-isotropic holomorphic curve in a complex quadric. Conversely, any 1-isotropic complex curve in this complex quadric describes a 2-parameter family of spheres whose envelope is always a Wintgen ideal submanifold of codimension two at the regular points. Via a complex stereographic projection, we show that our characterization is equivalent to Dajczer and Tojeiro's previous description of these submanifolds in terms of minimal surfaces in the Euclidean space.
\end{abstract}

1. Introduction. A remarkable result in the submanifold theory in real space forms is the so-called DDVV inequality, which relates the most important intrinsic and extrinsic quantities at an arbitrary point of a submanifold (like the scalar curvature, the mean curvature, and the scalar normal curvature), without any restriction on the dimension/codimension or any further geometric/topological assumptions. This universal inequality was a difficult conjecture in $[8,9]$, and was finally proved in [10] and [14].

It is very interesting to characterize the equality case in the DDVV inequality. By the suggestion of $[4,16]$ and the characterization of [10] about the equality case at an arbitrary point, we introduce the following definition.

DEFINITION 1.1. A submanifold $M^{m}$ of dimension $m$ and codimension $p$ in a real space form is called a Wintgen ideal submanifold if the equality is attained at every point of $M^{m}$. This happens if and only if at every point $x \in M$ there exists an orthonormal basis $\left\{e_{1}, \ldots, e_{m}\right\}$ of the tangent plane $T_{x} M^{m}$ and an orthonormal basis $\left\{n_{1}, \ldots, n_{p}\right\}$ of the normal

2010 Mathematics Subject Classification. Primary 53C42; Secondary 53A30, 53C43.

Key words and phrases. Wintgen ideal submanifolds, Möbius geometry, mean curvature sphere, conformal Gauss map, minimal surfaces, holomorphic curves.

The first author is supported by the Project No. 11571037 of NSFC. The corresponding author* is partially supported by the Project No. 10901006 and No. 11471021 of NSFC. The third author is partially supported by the Project No. 11331002 of NSFC. The fourth author is supported by the Fundamental Research Funds for the Central Universities. All authors are supported by the Project No. 11171004 of NSFC. 
plane $T_{x}^{\perp} M^{m}$, such that the shape operators $\left\{A_{n_{i}}, i=1, \ldots, p\right\}$ take the form as below [10]:

$$
\text { (1) } A_{n_{1}}=\left(\begin{array}{ccccc}
\lambda_{1} & \mu_{0} & 0 & \cdots & 0 \\
\mu_{0} & \lambda_{1} & 0 & \cdots & 0 \\
0 & 0 & \lambda_{1} & \cdots & 0 \\
\vdots & \vdots & \vdots & \ddots & \vdots \\
0 & 0 & 0 & \cdots & \lambda_{1}
\end{array}\right), A_{n_{2}}=\left(\begin{array}{ccccc}
\lambda_{2}+\mu_{0} & 0 & 0 & \cdots & 0 \\
0 & \lambda_{2}-\mu_{0} & 0 & \cdots & 0 \\
0 & 0 & \lambda_{2} & \cdots & 0 \\
\vdots & \vdots & \vdots & \ddots & \vdots \\
0 & 0 & 0 & \cdots & \lambda_{2}
\end{array}\right) \text {, }
$$

Note that the distribution $\mathbb{D}=\operatorname{Span}\left\{e_{1}, e_{2}\right\}$ is well-defined when the submanifold is umbilicfree. This is called the canonical distribution.

Wintgen ideal submanifolds are abundant. Wintgen [19] first proved the DDVV inequality for surfaces in $\mathbb{S}^{4}$. When the equality is attained everywhere, such surfaces are called super-conformal, which means that the curvature ellipse is a circle, or equivalently, the Hopf differential is an isotropic differential form. For more examples see [3, 5, 6, 7, 13, 20]. Generally a Wintgen ideal submanifold is not necessarily minimal; on the other hand, it is noteworthy that many important examples appearing in the partial classification results above come from holomorphic curves or minimal surfaces/submanifolds.

An important observation by Dajczer and Tojeiro [7] (based on the result in [9]) is that the DDVV inequality, as well as the equality case, is invariant under Möbius transformations of the ambient space. So it is clear that the most suitable framework for the study of Wintgen ideal submanifolds is Möbius geometry.

This research program has been carried out by us in [12] and [20]. In [12], we show that when the canonical distribution $\mathbb{D}$ generates a comparatively lower dimensional integrable distribution, a Wintgen ideal submanifold is a cylinder, a cone, or a rotational submanifold over a minimal Wintgen ideal submanifold in $\mathbb{R}^{n}, S^{n}$ or $H^{n}$, respectively. In [20], when the dimension $m=3$ and the codimension $p=2$, we show that $M^{3}$ has a circle bundle structure over a Riemann surface among other results. Observe that the sphere bundle structure manifests itself in both cases.

In this paper we concentrate on the codimension two case and consider an important Möbius invariant object associated with a submanifold $M$, the so-called mean curvature sphere. At each point $x \in M^{m}$, it is the unique $m$-dimensional round sphere tangent to $M^{m}$ at $x$ which also shares the same mean curvature vector with $M^{m}$ at $x$. In the codimension two case, this assigns a (oriented) space-like 2-space $\operatorname{Span}_{\mathbb{R}}\left\{\xi_{1}, \xi_{2}\right\}$ in the Lorentz space $\mathbb{L}^{m+4}$, which is also identified with the isotropic complex line $\operatorname{Span}_{\mathbb{C}}\left\{\xi_{1}-i \xi_{2}\right\} \in \mathbb{C} P^{m+3}$ (with respect to the $\mathbb{C}$-linear extension of the Lorentz metric). When the base point $x$ varies along $M^{m}$, we obtain the mean curvature sphere congruence, which is also represented as a Gauss map

$$
[\xi] \triangleq\left[\xi_{1}-i \xi_{2}\right]: M^{m} \rightarrow Q_{+}^{m+2}=\left\{[Z] \in \mathbb{C} P^{m+3} \mid\langle Z, Z\rangle=0,\langle Z, \bar{Z}\rangle>0\right\} .
$$

It is similar to the generalized Gauss map of a minimal surface in Euclidean space and the conformal Gauss map of a (Willmore) surface [1]. We call it the conformal Gauss map. 
The first key observation by us (see also [20]) is that under the hypothesis of being Wintgen ideal, this $m$-sphere congruence is indeed a 2-parameter family, and its envelope not only recovers $M^{m}$, but also extends it to a submanifold as a sphere bundle over a Riemann surface $\bar{M}^{2}$ (a holomorphic curve). The underlying surface $\bar{M}^{2}$ comes from the quotient surface $\bar{M}^{2}=M^{m} / \Gamma$ (at least locally) where $\Gamma$ is the foliation of $M^{m}$ by the integral submanifolds of the distribution $\mathbb{D}^{\perp}=\operatorname{Span}\left\{e_{3}, \ldots, e_{m}\right\}$. Moreover, the mean curvature sphere $\left[\xi_{1}-i \xi_{2}\right]$ indeed determines a holomorphic, 1 -isotropic curve in $Q_{+}^{m+2}$, and all codimension two Wintgen ideal submanifolds can be constructed by such curves in $Q_{+}^{m+2}$. The precise statement of our main result is as follows.

THEOREM 1.2. The mean curvature spheres $[\xi] \triangleq\left[\xi_{1}-i \xi_{2}\right] \in Q_{+}^{m+2}$ of a Wintgen ideal submanifold of codimension two is a holomorphic and 1-isotropic curve, i.e.,

$$
\xi_{\bar{z}} \| \xi,\left\langle\xi_{z}, \xi_{z}\right\rangle=0 .
$$

Conversely, given a holomorphic isotropic curve

$$
[\xi]: \bar{M}^{2} \rightarrow Q_{+}^{m+2} \subset \mathbb{C} P^{m+3},
$$

the envelope $\widehat{M}^{m}$ of the corresponding 2-parameter family spheres is an m-dimensional Wintgen ideal submanifold (at the regular points).

We would like to mention that this result can be generalized to the arbitrary codimensional case, yet only in a weaker form. When the codimension is larger than 2 , the mean curvature spheres still defines a conformal Gauss map into a Grassmannian, which is a conformal harmonic map (satisfying an additional isotropy condition). For details see another paper [15] by the second and the fourth authors.

This paper is organized as below. In Section 2 we give a brief review of the submanifold theory in Möbius geometry. In Section 3 we restrict to consider Wintgen ideal submanifolds of codimension two. The Möbius invariants now take a much simpler expression. As the core of this paper, in Section 4 we show that the mean curvature sphere congruence of a codimension two Wintgen ideal submanifold defines a 1-isotropic holomorphic curve in $Q_{+}^{m+2}$, and in Section 5 the converse is also proved. The geometry of such curves in $Q_{+}^{m+2}$ is also briefly explained in Section 5. These two parts finish the proof to the main theorem mentioned above.

It should be noted that Dajczer and Tojeiro already gave another description of Wintgen ideal submanifolds of codimension two in [7] via minimal surfaces in $\mathbb{R}^{m+2}$. Their construction is compared with ours in Section 6. Indeed, these two descriptions are equivalent by a correspondence between holomorphic, 1-isotropic curves in $Q_{+}^{m+2} \subset \mathbb{C} P_{1}^{m+3}$ (the conformal Gauss map of $M^{m}$ ) and those ones in $\mathbb{C}^{m+2}$ (the generalized Gauss map of minimal surfaces in $\mathbb{R}^{m+2}$ ). This comes from a correspondence between $Q^{m+2}$ and $\mathbb{C}^{m+2}$ which could be regarded as a complex version of the classical stereographic projection. This correspondence should have been well-known to experts. For example, see [2] where Bryant used algebraic geometry of complex curves in such a quadric $Q^{n}$ instead of studying holomorphic curves in $\mathbb{C}^{n}$ directly. 
In particular, we give a new proof to the following fact: When the ambient space is endowed with the Euclidean flat metric, the centers of the mean curvature spheres of a codimension two Wintgen ideal submanifold constitutes a minimal surface in this Euclidean space. This beautiful result was first obtained by Rouxel [17] for superconformal surfaces (i.e., Wintgen ideal surfaces) in $\mathbb{R}^{4}$, then re-discovered and generalized by Dajczer and Tojeiro $[6,7]$.

2. Basic invariants and equations for submanifolds in Möbius geometry. In this section we briefly review the theory of submanifolds in Möbius geometry. For details we refer to [18].

In the classical light-cone model, the light-like directions in the Lorentz space $\mathbb{R}_{1}^{m+p+2}$ correspond to points in the round sphere $\mathbb{S}^{m+p}$, and the Lorentz orthogonal group correspond to conformal transformation group of $\mathbb{S}^{m+p}$. The Lorentz inner product between $Y=$ $\left(Y_{0}, Y_{1}, \ldots, Y_{m+p+1}\right), Z=\left(Z_{0}, Z_{1}, \ldots, Z_{m+p+1}\right) \in \mathbb{R}_{1}^{m+p+2}$ is

$$
\langle Y, Z\rangle=-Y_{0} Z_{0}+Y_{1} Z_{1}+\cdots+Y_{m+p+1} Z_{m+p+1} .
$$

Let $x: M^{m} \rightarrow \mathbb{S}^{m+p} \subset \mathbb{R}^{m+p+1}$ be a submanifold without umbilics. Take $\left\{e_{i} \mid 1 \leq\right.$ $i \leq m\}$ as the tangent frame with respect to the induced metric $I=d x \cdot d x$, and $\left\{\theta_{i}\right\}$ as the dual 1-forms. Let $\left\{n_{r} \mid 1 \leq r \leq p\right\}$ be orthonormal frame for the normal bundle. The second fundamental form and the mean curvature of $x$ are

$$
I I=\sum_{i j, \gamma} h_{i j}^{r} \theta_{i} \otimes \theta_{j} n_{r}, \quad H=\frac{1}{m} \sum_{j, r} h_{j j}^{r} n_{r}=\sum_{r} H^{r} n_{r},
$$

respectively. We define the Möbius position vector $Y: M^{m} \rightarrow \mathbb{R}_{1}^{m+p+2}$ of $x$ by

$$
Y=\rho(1, x), \quad \rho^{2}=\frac{m}{m-1}\left|I I-\frac{1}{m} \operatorname{tr}(I I) I\right|^{2}
$$

which is also called the canonical lift of $x$ [18]. Two submanifolds $x, \bar{x}: M^{m} \rightarrow \mathbb{S}^{m+p}$ are Möbius equivalent if there exists $T$ in the Lorentz group $O(m+p+1,1)$ in $\mathbb{R}_{1}^{m+p+2}$ such that $\bar{Y}=Y T$. It follows immediately that

$$
\mathrm{g}=\langle d Y, d Y\rangle=\rho^{2} d x \cdot d x
$$

is a Möbius invariant, called the Möbius metric of $x$.

Let $\Delta$ be the Laplacian with respect to g. Define

$$
N=-\frac{1}{m} \Delta Y-\frac{1}{2 m^{2}}\langle\Delta Y, \Delta Y\rangle Y,
$$

which satisfies

$$
\langle Y, Y\rangle=0=\langle N, N\rangle,\langle N, Y\rangle=1 .
$$

Let $\left\{E_{1}, \ldots, E_{m}\right\}$ be a local orthonormal frame for $\left(M^{m}, \mathrm{~g}\right)$ with dual 1 -forms $\left\{\omega_{1}, \ldots, \omega_{m}\right\}$. Write $Y_{j}=E_{j}(Y)$. Then we have

$$
\left\langle Y_{j}, Y\right\rangle=\left\langle Y_{j}, N\right\rangle=0,\left\langle Y_{j}, Y_{k}\right\rangle=\delta_{j k}, \quad 1 \leq j, k \leq m .
$$


We define

$$
\xi_{r}=\left(H^{r}, n_{r}+H^{r} x\right) .
$$

Then $\left\{\xi_{1}, \ldots, \xi_{p}\right\}$ form the orthonormal frame of the orthogonal complement of $\operatorname{Span}\{Y, N$, $\left.Y_{j} \mid 1 \leq j \leq m\right\}$. And $\left\{Y, N, Y_{j}, \xi_{r}\right\}$ form a moving frame in $\mathbb{R}_{1}^{m+p+2}$ along $M^{m}$.

REMARK 2.1. Geometrically, at one point $x, \xi_{r}$ (for any given $r$ ) corresponds to the unique hyper-sphere tangent to $M_{m}$ with normal vector $n_{r}$ and mean curvature $H^{r}(x)$. In particular, the spacelike subspace $\operatorname{Span}_{\mathbb{R}}\left\{\xi_{1}, \ldots, \xi_{p}\right\}$ represents a unique $m$-dimensional sphere tangent to $M_{m}$ with the same mean curvature vector $\sum_{r} H^{r} n_{r}$. This well-defined object was naturally named the mean curvature sphere of $M^{m}$ at $x$. Note that it still share the same mean curvature at $x$ even when the ambient space is endowed with any other conformal metric.

We fix the range of indices in this section as below: $1 \leq i, j, k \leq m ; 1 \leq r, s \leq p$. The structure equations are:

$$
\begin{aligned}
& d Y=\sum_{i} \omega_{i} Y_{i}, \\
& d N=\sum_{i j} A_{i j} \omega_{i} Y_{j}+\sum_{i, r} C_{i}^{r} \omega_{i} \xi_{r}, \\
& d Y_{i}=-\sum_{j} A_{i j} \omega_{j} Y-\omega_{i} N+\sum_{j} \omega_{i j} Y_{j}+\sum_{j, r} B_{i j}^{r} \omega_{j} \xi_{r}, \\
& d \xi_{r}=-\sum_{i} C_{i}^{r} \omega_{i} Y-\sum_{i, j} \omega_{i} B_{i j}^{r} Y_{j}+\sum_{s} \theta_{r s} \xi_{s},
\end{aligned}
$$

where $\omega_{i j}$ are the connection 1-forms of the Möbius metric $\mathrm{g}$ and $\theta_{r s}$ the normal connection 1 -forms. The tensors

$$
\mathbf{A}=\sum_{i, j} A_{i j} \omega_{i} \otimes \omega_{j}, \quad \mathbf{B}=\sum_{i, j, r} B_{i j}^{r} \omega_{i} \otimes \omega_{j} \xi_{r}, \quad \Phi=\sum_{j, r} C_{j}^{r} \omega_{j} \xi_{r}
$$

are called the Blaschke tensor, the Möbius second fundamental form and the Möbius form of $x$, respectively. The covariant derivatives $A_{i j, k}, B_{i j, k}^{r}, C_{i, j}^{r}$ are defined as usual. For example,

$$
\begin{aligned}
& \sum_{j} C_{i, j}^{r} \omega_{j}=d C_{i}^{r}+\sum_{j} C_{j}^{r} \omega_{j i}+\sum_{s} C_{i}^{s} \theta_{s r} \\
& \sum_{k} B_{i j, k}^{r} \omega_{k}=d B_{i j}^{r}+\sum_{k} B_{i k}^{r} \omega_{k j}+\sum_{k} B_{k j}^{r} \omega_{k i}+\sum_{s} B_{i j}^{s} \theta_{s r} .
\end{aligned}
$$

The integrability conditions for the structure equations are given as below:

$$
\begin{aligned}
& A_{i j, k}-A_{i k, j}=\sum_{r} B_{i k}^{r} C_{j}^{r}-B_{i j}^{r} C_{k}^{r}, \\
& C_{i, j}^{r}-C_{j, i}^{r}=\sum_{k}\left(B_{i k}^{r} A_{k j}-B_{j k}^{r} A_{k i}\right), \\
& B_{i j, k}^{r}-B_{i k, j}^{r}=\delta_{i j} C_{k}^{r}-\delta_{i k} C_{j}^{r},
\end{aligned}
$$




$$
\begin{aligned}
R_{i j k l} & =\sum_{r} B_{i k}^{r} B_{j l}^{r}-B_{i l}^{r} B_{j k}^{r}+\delta_{i k} A_{j l}+\delta_{j l} A_{i k}-\delta_{i l} A_{j k}-\delta_{j k} A_{i l}, \\
R_{r s i j}^{\perp} & =\sum_{k} B_{i k}^{r} B_{k j}^{s}-B_{i k}^{s} B_{k j}^{r} .
\end{aligned}
$$

Here $R_{i j k l}$ denote the curvature tensor of $\mathrm{g}$. Other restrictions on the tensor $\mathbf{B}$ are

$$
\sum_{j} B_{j j}^{r}=0, \sum_{i, j, r}\left(B_{i j}^{r}\right)^{2}=\frac{m-1}{m} .
$$

All coefficients in the structure equations are determined by $\{\mathrm{g}, \mathbf{B}\}$ and the normal connection $\left\{\theta_{\alpha \beta}\right\}$. Coefficients of Möbius invariants and the isometric invariants are related as below. (We omit the formula for $A_{i j}$ since it will not be used later.)

$$
\begin{aligned}
B_{i j}^{r} & =\rho^{-1}\left(h_{i j}^{r}-H^{r} \delta_{i j}\right), \\
C_{i}^{r} & =-\rho^{-2}\left[H_{, i}^{r}+\sum_{j}\left(h_{i j}^{r}-H^{r} \delta_{i j}\right) e_{j}(\ln \rho)\right] .
\end{aligned}
$$

REMARK 2.2. For $x: M^{m} \rightarrow \mathbb{R}^{m+p}$, the Möbius position vector $Y: M^{m} \rightarrow \mathbb{R}_{1}^{m+p+2}$ and the mean curvature sphere $\left\{\xi_{1}, \ldots, \xi_{p}\right\}$ are given by

$$
\begin{gathered}
Y=\rho\left(\frac{1+|x|^{2}}{2}, \frac{1-|x|^{2}}{2}, x\right), \\
\xi_{r}=\left(\frac{1+|x|^{2}}{2}, \frac{1-|x|^{2}}{2}, x\right) H^{r}+\left(x \cdot n_{r},-x \cdot n_{r}, n_{r}\right) .
\end{gathered}
$$

3. Wintgen ideal submanifolds of codimension 2. From now on, we assume $x$ : $M^{m} \rightarrow \mathbb{S}^{m+2}$ to be a codimension two Wintgen ideal submanifold. According to [7] and [10], that means we can choose a suitable tangent and normal frame $\left(\left\{E_{1}, \ldots, E_{m}\right\}\right.$ and $\left.\left\{\xi_{1}, \xi_{2}\right\}\right)$ such that the Möbius second fundamental form $B$ can be written down as follows:

$$
B^{1}=\left(\begin{array}{ccccc}
0 & \mu & 0 & \cdots & 0 \\
\mu & 0 & 0 & \cdots & 0 \\
0 & 0 & 0 & \cdots & 0 \\
\vdots & \vdots & \vdots & \ddots & \vdots \\
0 & 0 & 0 & \cdots & 0
\end{array}\right), B^{2}=\left(\begin{array}{ccccc}
\mu & 0 & 0 & \cdots & 0 \\
0 & -\mu & 0 & \cdots & 0 \\
0 & 0 & 0 & \cdots & 0 \\
\vdots & \vdots & \vdots & \ddots & \vdots \\
0 & 0 & 0 & \cdots & 0
\end{array}\right), \quad \mu=\sqrt{\frac{m-1}{4 m}}
$$

Note that $\mu=\sqrt{\frac{m-1}{4 m}}$ is a constant determined by (13). The canonical distribution $\mathbb{D}=$ $\operatorname{Span}\left\{E_{1}, E_{2}\right\}$ is well-defined, as well as its orthogonal distribution $\mathbb{D}^{\perp}=\operatorname{Span}\left\{E_{3}, \ldots, E_{m}\right\}$.

For convenience we adopt the convention below on the range of indices:

$$
1 \leq i, j, k, l \leq m, \quad 3 \leq a, b \leq m .
$$


First we compute the covariant derivatives of $B_{i j}^{r}$. The result is

$$
\begin{aligned}
& \sum_{j} B_{11, j}^{1} \omega_{j}=-\mu \theta, \sum_{j} B_{11, j}^{2} \omega_{j}=0, \\
& \sum_{j} B_{12, j}^{1} \omega_{j}=0, \quad \sum_{j} B_{12, j}^{2} \omega_{j}=\mu \theta \\
& \sum_{j} B_{22, j}^{1} \omega_{j}=\mu \theta, \quad \sum_{j} B_{22, j}^{2} \omega_{j}=0
\end{aligned}
$$

and

$$
\begin{aligned}
& \sum_{j} B_{1 a, j}^{1} \omega_{j}=\mu \omega_{2 a}, \quad \sum_{j} B_{1 a, j}^{2} \omega_{j}=\mu \omega_{1 a}, \\
& \sum_{j} B_{2 a, j}^{1} \omega_{j}=\mu \omega_{1 a}, \quad \sum_{j} B_{2 a, j}^{2} \omega_{j}=-\mu \omega_{2 a}, \\
& B_{a b, j}^{1}=0, B_{a b, j}^{2}=0 .
\end{aligned}
$$

Here

$$
\theta \triangleq 2 \omega_{12}+\theta_{12}
$$

is a combination of the connection 1-forms of the bundle $\mathbb{D}$ and the normal bundle.

Since $B_{i j, k}^{r}$ is symmetric on distinct $i, j, k$ by (10), using (17) and (18), we have

$$
B_{1 a, 2}^{1}=B_{2 a, 1}^{1}=B_{12, a}^{1}=0 ; \quad B_{1 a, b}^{1}=B_{2 a, b}^{1}=0(\text { if } a \neq b) .
$$

Again by (18) we obtain

$$
\begin{aligned}
& \omega_{1 a}=\frac{1}{\mu} \sum_{j} B_{2 a, j}^{1} \omega_{j}=\frac{1}{\mu}\left(B_{2 a, 2}^{1} \omega_{2}+B_{2 a, a}^{1} \omega_{a}\right)=\frac{1}{\mu} \sum_{j} B_{1 a, j}^{2} \omega_{j}, \\
& \omega_{2 a}=\frac{1}{\mu} \sum_{j} B_{1 a, j}^{1} \omega_{j}=\frac{1}{\mu}\left(B_{1 a, 1}^{1} \omega_{1}+B_{1 a, a}^{1} \omega_{a}\right)=-\frac{1}{\mu} \sum_{j} B_{2 a, j}^{2} \omega_{j} .
\end{aligned}
$$

Comparing the components above implies

$$
B_{1 a, 1}^{2}=B_{2 a, 2}^{2}=0 .
$$

By (18), $B_{11, a}^{2}=B_{22, a}^{2}=0$. Together with (10), (23) we get that for $3 \leq a \leq m$,

$$
\begin{aligned}
C_{a}^{2} & =B_{11, a}^{2}-B_{1 a, 1}^{2}=0 ; \\
C_{a}^{1} & =B_{11, a}^{1}-B_{1 a, 1}^{1}=-\mu \theta\left(e_{3}\right)-\mu \omega_{23}\left(e_{1}\right) \\
& =B_{22, a}^{1}-B_{2 a, 2}^{1}=\mu \theta\left(e_{3}\right)-\mu \omega_{13}\left(e_{2}\right) .
\end{aligned}
$$


Similarly there is

$$
\begin{aligned}
& C_{1}^{1}=B_{22,1}^{1}=\mu \theta\left(e_{1}\right)=-B_{1 a, a}^{1}=-\mu \omega_{2 a}\left(e_{a}\right), \\
& C_{2}^{1}=B_{11,2}^{1}=-\mu \theta\left(e_{2}\right)=-B_{2 a, a}^{1}=-\mu \omega_{1 a}\left(e_{a}\right), \\
& C_{1}^{2}=-B_{12,2}^{2}=-\mu \theta\left(e_{2}\right)=-B_{1 a, a}^{2}=-\mu \omega_{1 a}\left(e_{a}\right), \\
& C_{2}^{2}=-B_{12,1}^{2}=-\mu \theta\left(e_{1}\right)=-B_{2 a, a}^{2}=\mu \omega_{2 a}\left(e_{a}\right) .
\end{aligned}
$$

In particular we have

$$
C_{1}^{1}=-C_{2}^{2}, C_{2}^{1}=C_{1}^{2} .
$$

Also note that the normal connection 1-form $\theta_{12}=-\theta_{21}$. Substitute these relations into the last structure equation in (6). We obtain

$$
\begin{aligned}
& d \xi_{1}=-\left(C_{1}^{1} \omega_{1}+C_{2}^{1} \omega_{2}\right) Y-\mu\left(\omega_{1} Y_{2}+\omega_{2} Y_{1}\right)+\theta_{12} \xi_{2}, \\
& d \xi_{2}=-\left(C_{2}^{1} \omega_{1}-C_{1}^{1} \omega_{2}\right) Y-\mu\left(\omega_{1} Y_{1}-\omega_{2} Y_{2}\right)-\theta_{12} \xi_{1} .
\end{aligned}
$$

Combining these two equations and re-writing them using the complexified frame, we obtain an elegant formula as below:

$$
d\left(\xi_{1}-i \xi_{2}\right)=i \mu\left(\omega_{1}+i \omega_{2}\right)\left(\eta_{1}+i \eta_{2}\right)+i \theta_{12}\left(\xi_{1}-i \xi_{2}\right)
$$

where

$$
\eta_{1}=Y_{1}+\frac{C_{2}^{1}}{\mu} Y, \quad \eta_{2}=Y_{2}+\frac{C_{1}^{1}}{\mu} Y .
$$

This formula and its geometric explanation is the focus of this paper.

As a preparation, we point out that for a codimension two submanifold in $\mathbb{S}^{m+2}$, the mean curvature sphere defines the conformal Gauss map into the Grassmann manifold $\operatorname{Gr}\left(2, \mathbb{R}_{1}^{m+4}\right)$, the module space of spacelike 2-planes in the Lorentz space which is a pseudo-Riemannian symmetric space. This can be identified with a non-compact complex quadric $Q_{+}^{m+2} \subset$ $\mathbb{C} P^{m+3}$ via the following correspondence

$$
\operatorname{Span}_{\mathbb{R}}\left\{\xi_{1}, \xi_{2}\right\} \leftrightarrow\left[\xi_{1}-i \xi_{2}\right] \in \mathbb{C} P^{m+3} .
$$

4. The geometry of 1-isotropic complex curves in $Q_{+}^{m+2}$. To describe $Q_{+}^{m+2}$, note that the complex space $\mathbb{C}_{1}^{m+4}=\mathbb{R}_{1}^{m+4} \otimes \mathbb{C}$ is endowed with the complex inner product coming from the bilinear extension of the Lorentz metric. The null lines in this space define an $m+2$ dimensional compact complex quadric hypersurface

$$
Q^{m+2}=\left\{[\xi] \in \mathbb{C} P^{m+3} \mid \xi \in \mathbb{C}_{1}^{m+4},\langle\xi, \xi\rangle=0\right\} .
$$

This $\xi$ is either a complex multiple of a light-like vector in $\mathbb{R}_{1}^{m+4}$, or it can be written as $\xi=\xi_{1}-i \xi_{2}$ where $\left\{\xi_{1}, \xi_{2}\right\}$ is an orthonormal frame of a spacelike 2-space.

In the first case, such [ $\xi$ ]'s form the projective light cone which could be identified with the sphere $\mathbb{S}^{m+2}$.

In the second case, they form the quadric

$$
Q_{+}^{m+2}=\{[\xi] \mid\langle\xi, \xi\rangle=0,\langle\xi, \bar{\xi}\rangle>0\} \cong Q^{m+2} \backslash \mathbb{S}^{m+2}
$$


which is a non-compact complex manifold endowed with an indefinite Hermitian metric with signature $(m+1,1)$. In terms of the local lift $\xi$, this Hermitian metric is defined by

$$
h_{\xi}=\frac{1}{\langle\xi, \bar{\xi}\rangle}\left\langle d \xi-\frac{\langle d \xi, \bar{\xi}\rangle}{\langle\xi, \bar{\xi}\rangle} \xi, d \bar{\xi}-\frac{\langle d \bar{\xi}, \xi\rangle}{\langle\xi, \bar{\xi}\rangle} \bar{\xi}\right\rangle \text {. }
$$

It is evident that this metric is independent to the choice of the lift $\xi$, and it is invariant under the action of the Lorentz orthogonal group $O(m+3,1)$.

A complex curve in $Q_{+}^{m+2}$ is a holomorphic immersion of a Riemann surface [ $\left.\xi\right]: \bar{M}^{2} \rightarrow$ $Q_{+}^{m+2}$, given by a local lift $\xi: \bar{M}^{2} \rightarrow \mathbb{C}_{1}^{m+4}$ satisfying

$$
\frac{\partial}{\partial \bar{z}} \xi=\lambda \xi \| \xi, \text { where } \frac{\partial}{\partial \bar{z}} \triangleq \frac{1}{2}\left(\frac{\partial}{\partial u}+i \frac{\partial}{\partial v}\right)
$$

for some local complex function $\lambda$ and local complex coordinate $z=u+i v$ of $\bar{M}^{2}$.

A complex curve $[\xi]: \bar{M}^{2} \rightarrow Q_{+}^{m+2}$ is called 1-isotropic if and only if the complex differential $d \xi$ is isotropic.

The following is a characterization of such holomorphic 1-isotropic curves. The easy proof is omitted at here.

LEMMA 4.1. A map $\xi=\xi_{1}-i \xi_{2}$ from a Riemann surface $\bar{M}^{2}$ to $\mathbb{C}_{1}^{m+4}$ determines a 1-isotropic and holomorphic immersion $[\xi]: \bar{M}^{2} \rightarrow Q_{+}^{m+2}$ if and only if $\langle\xi, \xi\rangle=0$ and the horizontal part

$$
d \xi-\frac{\langle d \xi, \bar{\xi}\rangle}{\langle\xi, \bar{\xi}\rangle} \xi
$$

is a vector-valued $(1,0)$ form which is isotropic.

In other words, locally there exist $(1,0)$ form $\theta_{1}+i \theta_{2}, 1$-form $\theta_{12}$ and orthonormal frame vectors $\xi_{1}, \xi_{2}, \eta_{1}, \eta_{2} \in \mathbb{R}_{1}^{m+4}$ on $\bar{M}^{2}$ such that

$$
d \xi=i \mu\left(\theta_{1}+i \theta_{2}\right)\left(\eta_{1}+i \eta_{2}\right)+i \theta_{12} \xi, \mu=\sqrt{\frac{m-1}{4 m}} .
$$

5. Mean curvature spheres correspond to a holomorphic 1-isotropic curve. In this section, we will show that the mean curvature sphere congruence of a Wintgen ideal submanifold $M^{m} \rightarrow \mathbb{S}^{m+2}$ is a 2-parameter family of $m$-spheres. Moreover, this conformal Gauss map defines a holomorphic, 1-isotropic curve in $Q_{+}^{m+2}$, and the submanifold can be recovered from this curve.

THEOREM 5.1. For a Wintgen ideal submanifold $x: M^{m} \rightarrow \mathbb{S}^{m+2}$ which is umbilicfree we have:

(1) The complex vector-valued function $\xi=\xi_{1}-i \xi_{2}$ defines the conformal Gauss map

$$
[\xi]: \bar{M}^{2} \rightarrow Q_{+}^{m+2} \subset \mathbb{C} P_{1}^{m+3} \text {. }
$$

The image is a 1-isotropic complex curve in the sense that

$$
\langle d \xi, d \xi\rangle=0,\langle d \xi, d \bar{\xi}\rangle>0 .
$$


(2) The distribution $\mathbb{D}^{\perp}=\operatorname{Span}\left\{E_{3}, \ldots, E_{m}\right\}$ is integrable. Its integral submanifolds define a foliation $\mathscr{D}$ of $M^{m}$. Moreover, we have the quotient manifold structure

$$
\bar{M}^{2}=M^{m} / \mathscr{D} \text {. }
$$

(3) The projection $\pi: M^{m} \rightarrow \bar{M}^{2}$ is a Riemannian submersion (up to the factor $\mu$ ), where $M^{m}$ is endowed with the Möbius metric and $\bar{M}^{2}$ is endowed with the induced metric from $Q_{+}^{m+2}$.

(4) The mean curvature spheres $\operatorname{Span}\left\{\xi_{1}, \xi_{2}\right\}$ is a 2-parameter family. They envelope an $m$-dimensional submanifold $\widehat{M}^{m} \supset M^{m}$ (it might degenerate at some points).

(5) $\mathscr{D}$ extends to the whole envelope $\widehat{M}^{m}$ as a foliation by a 2-parameter family of $(m-2)$ dimensional spheres. In other words, $\widehat{M}^{m}$ can be viewed as a sphere bundle over the Riemann surface $\bar{M}^{2}$.

Proof. By the assumption that $M^{m}$ is Wintgen ideal with codimension two, we have obtained the formula (27):

$$
d\left(\xi_{1}-i \xi_{2}\right)=i \mu\left(\omega_{1}+i \omega_{2}\right)\left(\eta_{1}+i \eta_{2}\right)+i \theta_{12}\left(\xi_{1}-i \xi_{2}\right),
$$

with $\eta_{1}=Y_{1}+\frac{C_{2}^{1}}{\mu} Y, \eta_{2}=Y_{2}+\frac{C_{1}^{1}}{\mu} Y$. It follows that the tangent map of [ $\left.\xi\right]$ maps the tangent space $T_{p} M^{m}$ at one point $p \in M^{m}$ to a 1-dimensional complex line in the tangent space of $T Q_{+}^{m+2}$ at the corresponding image point. Thus the image of $M^{m}$ under this map is a complex curve of $Q_{+}^{m+2}$. Intrinsically this is a Riemann surface, which we denote as $\bar{M}^{2}$. This proves the first part of the conclusion (1).

It is clear from (21) and (22) that the distribution

$$
\mathbb{D}^{\perp}=\operatorname{Span}\left\{E_{3}, \ldots, E_{m}\right\}
$$

is integrable. These $(m-2)$-dimensional integral submanifolds of $\mathbb{D}^{\perp}$ defines a foliation $\mathscr{D}$ of $M$. Along each leave of $\mathscr{D}$, the restriction of the tangent map $d \xi$ is parallel to $\xi$ by (27). So $[\xi]: M^{m} \rightarrow Q_{+}^{m+2}$ is always constant when restricted to such a leave. This enables us to define a quotient map

$$
M^{m} \rightarrow M^{m} / \mathscr{D} \cong \bar{M}^{2}
$$

where each leave of $\mathscr{D}$ is mapped to a single point. In particular this is a submersion between differentiable manifolds. By (27) this is even a Riemannian submersion up to the factor $\mu$. Thus the conclusion (2) and (3) are established.

With respect to the induced Riemann surface structure and local complex coordinate $z$, $d z$ should be a multiple of $\omega_{1}+i \omega_{2}$. Regard $d \xi$ as a vector-valued complex differential form, it follows from (27) that $\langle d \xi, d \xi\rangle=0,\langle d \xi, d \bar{\xi}\rangle>0$. So the conclusion (1) has been proved completely.

By the conclusion (1), the mean curvature sphere congruence $\operatorname{Span}\left\{\xi_{1}, \xi_{2}\right\}$ is obviously a real 2-parameter family. In Möbius geometry, it is well-known that such a sphere congruence has an envelope if and only if $\xi_{1}, \xi_{2}, d \xi_{1}, d \xi_{2}$ always span a family of 4-dimensional spacelike subspaces, and the points on the envelope is given by the light-like directions located in 
the orthogonal complements of $\operatorname{Span}\left\{\xi_{1}, \xi_{2}, d \xi_{1}, d \xi_{2}\right\}$. According to (27), this is exactly such a case. We denote the envelope as $\widehat{M}^{m} \supset M^{m}$, which consists of a 2-parameter family of $(m-2)$-dimensional spheres; each $(m-2)$-dimensional sphere corresponds to the orthogonal complement of $\operatorname{Span}_{\mathbb{R}}\left\{\xi_{1}, \xi_{2}, \eta_{1}, \eta_{2}\right\}_{p}$ at one point $p \in M^{m}$. This establishes the conclusion (4).

We assert that every integral submanifold of $\mathbb{D}^{\perp}$ in $M^{m}$ is contained in such an $(m-2)$ dimensional sphere. To see that, take exterior differentiation at both sides of (27). The result looks like

$$
d\left(\eta_{1}+i \eta_{2}\right)=\left(\omega_{1}+i \omega_{2}\right) \cdot \eta+(\cdots)\left(\eta_{1}+i \eta_{2}\right)+(\cdots)\left(\xi_{1}-i \xi_{2}\right),
$$

where the component $\eta$ is orthogonal to $\operatorname{Span}_{\mathbb{C}}\left\{\xi_{1}, \xi_{2}, d \xi_{1}, d \xi_{2}\right\}$. It follows that the subspace $V=\operatorname{Span}_{\mathbb{R}}\left\{\xi_{1}, \xi_{2}, \eta_{1}, \eta_{2}\right\}$ is invariant along any leave of the foliation $\mathscr{D}$. In particular, the integration of $Y$ along $\mathscr{D}$ is always located in the orthogonal complement of $V$, which implies that any integral submanifold is located on the corresponding $(m-2)$-dimensional sphere. So we obtain the conclusion (5).

In particular, this shows that the foliation structure $\mathscr{D}$ of $M^{m}$ is indeed an $(m-2)$ dimensional sphere bundle over a Riemann surface $\bar{M}^{2}$. This finishes the proof.

In the statement of the theorem above, we can add that the envelope $\widehat{M}^{m} \supset M^{m}$ is still a Wintgen ideal submanifold (on the subset where it is an immersed submanifold). This is the corollary of the next theorem, which is the converse of Theorem 5.1.

THEOREM 5.2. Given a holomorphic, isotropic curve $[\xi]: \bar{M}^{2} \rightarrow \mathbb{Q}$. The envelope $\widehat{M}^{m}$ of the corresponding 2-parameter family spheres is an m-dimensional Wintgen ideal submanifold when it is immersed, and $\widehat{M}^{m}$ has $[\xi]$ as its mean curvature sphere.

PROOF. The proof is a little bit long, so a sketch might be helpful. First we will give a local description of the envelope $\widehat{M}^{m}$ as an immersion $\hat{Y}: U \times S^{m-2} \rightarrow \mathbb{S}^{m+2}$ for $U \subset \bar{M}$. After that we will introduce a moving frame along $U \times S^{m-2}$ and write out the structure equations. The crucial step is to show that $\hat{Y}$ still has $[\xi]$ as its mean curvature sphere. Then it is straightforward to see that $\hat{Y}$ is Wintgen ideal.

By Lemma 4.1, the assumption of being holomorphic and isotropic implies

$$
d\left(\xi_{1}-i \xi_{2}\right)=i \mu\left(\theta_{1}+i \theta_{2}\right)\left(\eta_{1}+i \eta_{2}\right)+i \theta_{12}\left(\xi_{1}-i \xi_{2}\right),
$$

where $\mu=\sqrt{\frac{m-1}{4 m}}, \theta_{1}, \theta_{2}, \theta_{12}$ are real 1-forms locally defined on the underlying Riemann surface $\bar{M}$.

It follows that $\left\{\xi_{1}, \xi_{2}, d \xi_{1}, d \xi_{2}\right\}$ span a 4-dimensional spacelike subspace $V$. So the sphere congruence $\operatorname{Span}_{\mathbb{R}}\left\{\xi_{1}, \xi_{2}\right\}$ has an envelope $\widehat{M}$ which consists of the light-like directions in the orthogonal complement $V^{\perp}$. Locally we can restrict to a small neighborhood $U \subset \bar{M}^{2}$ and choose smoothly local pseudo-orthonormal frames at every point $q \in U$

$$
e_{0}(q), e_{1}(q), \ldots, e_{m-1}(q) \in V^{\perp}(q),\left\langle e_{0}(q), e_{0}(q)\right\rangle=-1 .
$$


Then one may parameterize $\widehat{M}^{m}$ explicitly as $U \times S^{m-2} \rightarrow \mathbb{S}^{m+2}$ given by

$$
(q, \Theta) \mapsto[\hat{Y}]=\left[e_{0}(q)+\sum_{j=1}^{m-1} \Theta_{j} e_{j}(q)\right]
$$

for $q \in U$ and $\Theta=\left(\Theta_{1}, \ldots, \Theta_{m-1}\right) \in S^{m-2}$ the coordinates of a unit sphere in $(m-1)$ dimensional Euclidean space. We want to show that this is a Wintgen ideal submanifold if it is immersed.

We introduce a moving frame along $U \times S^{m-2}$ :

$$
\left\{\hat{Y}, Y, \eta_{3}, \ldots, \eta_{m}\right\} \perp\left\{\eta_{1}, \eta_{2}, \xi_{1}, \xi_{2}\right\}
$$

it is required to be orthonormal except that

$$
\langle Y, Y\rangle=0=\langle\hat{Y}, \hat{Y}\rangle,\langle Y, \hat{Y}\rangle=1 .
$$

We emphasize that the frame vectors $\left\{\eta_{1}, \eta_{2}, \xi_{1}, \xi_{2}\right\}$ are now defined on $U \times S^{m-2}$ by extending them as constants along the fiber $S^{m-2}$. We will also abuse the notation $\theta_{1}, \theta_{2}, \theta_{12}$ to mean their pull-back to $U \times S^{m-2} \subset \widehat{M}$ under the natural projection map. Then (31) is still valid under this understanding. Using this moving frame, we write down the structure equations (the convention on the range of indices is $1 \leq j, k \leq m, 3 \leq a, b \leq m$ ):

$$
\begin{aligned}
d \xi_{1} & =-\mu \theta_{2} \eta_{1}-\mu \theta_{1} \eta_{2}+\theta_{12} \xi_{2} \\
d \xi_{2} & =-\mu \theta_{1} \eta_{1}+\mu \theta_{2} \eta_{2}-\theta_{12} \xi_{1} \\
d \eta_{1} & =-\hat{\omega}_{1} Y-\omega_{1} \hat{Y}+\sum_{k} \Omega_{1 k} \eta_{k}+\mu \theta_{2} \xi_{1}+\mu \theta_{1} \xi_{2} \\
d \eta_{2} & =-\hat{\omega}_{2} Y-\omega_{2} \hat{Y}+\sum_{k} \Omega_{2 k} \eta_{k}+\mu \theta_{1} \xi_{1}-\mu \theta_{2} \xi_{2} \\
d \eta_{a} & =-\hat{\omega}_{a} Y-\omega_{a} \hat{Y}+\sum_{k} \Omega_{a k} \eta_{k} \\
d Y & =\omega Y+\omega_{1} \eta_{1}+\omega_{2} \eta_{2}+\sum_{a} \omega_{a} \eta_{a} \\
d \hat{Y} & =-\omega \hat{Y}+\hat{\omega}_{1} \eta_{1}+\hat{\omega}_{2} \eta_{2}+\sum_{a} \hat{\omega}_{a} \eta_{a} .
\end{aligned}
$$

Here $\omega, \omega_{k}, \hat{\omega}_{k}, \Omega_{j k}$ are 1-forms locally defined on $U \times S^{m-2} \subset \widehat{M}$ which we don't need to know explicitly.

On the other hand, the coefficients of $\xi_{1}, \xi_{2}$ in these equations are explicitly determined by (32), (33) and the orthogonality of the frames.

The crucial observation is that there exist some functions $\hat{F}, \hat{G}$ such that

$$
\hat{\omega}_{1}=\hat{F} \theta_{1}+\hat{G} \theta_{2}, \hat{\omega}_{2}=-\hat{G} \theta_{1}+\hat{F} \theta_{2} .
$$

This follows from differentiating (38) and comparing the coefficients of $\xi_{1}, \xi_{2}$; or equivalently, by comparison between (30) and (34), (35). In particular,

$$
\hat{\omega}_{1}+i \hat{\omega}_{2}=(\hat{F}-i \hat{G})\left(\theta_{1}+i \theta_{2}\right) .
$$

Now we turn to the key observation as below. 
CLAIM. The submanifold $[\hat{Y}]$ has $\operatorname{Span}_{\mathbb{R}}\left\{\xi_{1}, \xi_{2}\right\}$ as its mean curvature sphere.

To show this, under the induced metric $\langle d \hat{Y}, d \hat{Y}\rangle=\sum_{j=1}^{m} \hat{\omega}_{j}^{2}$ we take the orthonormal dual frame $\left\{\hat{E}_{j}\right\}_{j=1}^{m}$. Then one can compute the Laplacian $\hat{\Delta} \hat{Y}$ so that we can determine the normal frames of $\hat{Y}$. Because the mean curvature sphere is determined by the subspace

$$
\operatorname{Span}_{\mathbb{R}}\left\{\hat{Y}, \hat{Y}_{j}, \sum_{j=1}^{m} \hat{E}_{j} \hat{E}_{j}(\hat{Y})\right\}=\operatorname{Span}_{\mathbb{R}}\left\{\hat{Y}, \hat{Y}_{j}, \hat{\Delta} \hat{Y}\right\},
$$

it suffices to show $\left\langle\sum_{j=1}^{m} \hat{E}_{j} \hat{E}_{j}(\hat{Y}), \xi_{1}-i \xi_{2}\right\rangle=0$, or equivalently,

$$
\left\langle\hat{Y}, \sum_{j=1}^{m} \hat{E}_{j} \hat{E}_{j}\left(\xi_{1}-i \xi_{2}\right)\right\rangle=0 \text {. }
$$

This is because $\left\langle\hat{Y}, \xi_{r}\right\rangle=0=\left\langle d \hat{Y}, \xi_{r}\right\rangle=\left\langle\hat{Y}, d \xi_{r}\right\rangle$. By (31) and (40), we have

$$
\sum_{j=1}^{m} \hat{E}_{j} \hat{E}_{j}\left(\xi_{1}-i \xi_{2}\right) \equiv \sum_{j=1}^{2} \hat{E}_{j} \hat{E}_{j}\left(\xi_{1}-i \xi_{2}\right) \equiv\left(\hat{E}_{1}-i \hat{E}_{2}\right)\left(\hat{E}_{1}+i \hat{E}_{2}\right)\left(\xi_{1}-i \xi_{2}\right)=0,
$$

where the equalities are up to some components orthogonal to $\hat{Y}$. This completes the proof of the previous claim.

Finally, for $\hat{Y}$ we take its canonical lift, whose derivatives are clear to be combinations of $\hat{Y}, \eta_{1}, \eta_{2}, \eta_{a}$. Its normal frame is just $\left\{\xi_{1}, \xi_{2}\right\}$ as we have shown. One reads from (32) and (33) that its Möbius second fundamental form still take the same form as (16). Thus it is a Wintgen ideal submanifold.

REMARK 5.3. In the proof above, one can choose a scaling of $\hat{Y}$ suitably so that $\hat{F}^{2}+$ $\hat{G}^{2}=1$. Then one can verify that this $\hat{Y}$ is exactly the canonical lift. It is straightforward to check that the projection

$$
\mathbb{S}^{m+2} \ni[\hat{Y}](p) \mapsto \operatorname{Span}_{\mathbb{R}}\left\{\xi_{1}, \xi_{2}\right\} \in \operatorname{Gr}\left(2, \mathbb{R}_{1}^{m+4}\right)
$$

is a Riemannian submersion (up to the factor $\mu=\sqrt{\frac{m-1}{4 m}}$ ) from $\widehat{M}^{m}$ to the Riemann surface $\bar{M}^{2}$. This agrees with the conclusion (3) of Theorem 5.2.

6. Relationship with minimal surfaces in $\mathbb{R}^{m+2}$. Dajczer and Tojeiro [6, 7] described another construction of almost all codimension two Wintgen ideal submanifolds via minimal surfaces in $\mathbb{R}^{m+2}$. The following theorem gives a nice geometric correspondence from Wintgen ideal submanifolds to Euclidean minimal surfaces. This result was obtained explicitly in [17,6] and implicitly contained in the main theorem of [7].

THEOREM 6.1. Given a Wintgen ideal submanifold $x: M^{m} \rightarrow \mathbb{R}^{m+2}$. Then the centers of its mean curvature sphere congruence form a two dimensional submanifold immersed in $\mathbb{R}^{m+2}$ which is a Euclidean minimal surface. 
REMARK 6.2. It is noteworthy that the statement of Theorem 6.1 involves some kind of symmetry breaking. The Wintgen ideal property and the mean curvature spheres are Möbius invariant. On the other hand, the centers of those spheres as well as the minimal property depends on a choice of the ambient space metric.

To clarify this problem, consider a given Wintgen ideal submanifold $x: M^{m} \rightarrow \mathbb{S}^{m+2}$. Assign an arbitrary point $p \in \mathbb{S}^{m+2} \subset \mathbb{R}^{m+3}$ as the north pole, $p=(1,0, \ldots, 0)$ up to a choice of the coordinate system. Then take the stereographic projection

$$
\mathbb{R}^{m+3} \supset \mathbb{S}^{m+2} \backslash\{p\} \ni x=\left(x^{\prime}, \vec{x}^{\prime \prime}\right) \rightarrow \frac{\vec{x}^{\prime \prime}}{1-x^{\prime}} \in \mathbb{R}^{m+2}, \quad x^{\prime} \in \mathbb{R}, \vec{x}^{\prime \prime} \in \mathbb{R}^{m+2} .
$$

Since this is a conformal diffeomorphism, the image is still a Wintgen ideal submanifold, and the mean curvature spheres are mapped to mean curvature spheres. These $m$-spheres is a 2-parameter family according to Theorem 5.1. Under this circumstance, Theorem 6.1 is equivalent to saying that the centers of these spheres constitute a minimal surface in this ambient flat space. Moreover, no matter which $p \in \mathbb{S}^{m+2}$ is chosen to be $\infty$ (the point at infinity of an affine space $\mathbb{V}_{p}^{m+2}$ ), the corresponding locus of the centers of those mean curvature spheres is always a minimal surface in this $\mathbb{V}_{p}^{m+2}$.

Below we provide a new proof to Theorem 6.1 according to the understanding of Remark 6.2.

Proof to TheOREM 6.1. Assign an arbitrary point $p=[\wp] \in \mathbb{S}^{m+2}$ to be the point at infinity, represented by the light-like vector

$$
\wp=(1,1, \overrightarrow{0}), \quad \overrightarrow{0} \in \mathbb{R}^{m+2} .
$$

Let $x: M^{m} \rightarrow \mathbb{S}^{m+2}$ be a Wintgen ideal submanifold with conformal Gauss map $[\xi]=$ $\left[\xi_{1}-i \xi_{2}\right]$. Without loss of generality we may suppose that locally these mean curvature spheres do not pass through $p$, or equivalently, that $\langle\xi, \wp\rangle \neq 0$. In the Euclidean space

$$
\mathbb{V}_{p}^{m+2} \cong \mathbb{S}^{m+2} \backslash\{p\}
$$

the center of the mean curvature sphere $\operatorname{Span}_{\mathbb{R}}\left\{\xi_{1}, \xi_{2}\right\}$ is nothing but the inversive image of $p$ with respect to this round sphere. In the light-cone model, the center $\left[O_{\xi}\right]$ is the image of $[\wp]$ under the reflection with respect to the subspace $\operatorname{Span}_{\mathbb{R}}\left\{\xi_{1}, \xi_{2}\right\}$. This is written down explicitly as

$$
O_{\xi}=\wp-2\left\langle\wp, \xi_{1}\right\rangle \xi_{1}-2\left\langle\wp, \xi_{2}\right\rangle \xi_{2}=\wp-\langle\wp, \xi\rangle \bar{\xi}-\langle\wp, \bar{\xi}\rangle \xi,
$$

where $\bar{\xi}=\xi_{1}+i \xi_{2}$ is the complex conjugation of $\xi$.

To show that this is a minimal surface in $\mathbb{V}_{p}^{m+2}$, we need to write down the mapping to $\mathbb{R}^{m+2}$ explicitly. We re-write the classical stereographic projection (41) as from the projective lightcone to $\mathbb{R}^{m+2}$ :

$$
[\tilde{x}]=\left[\frac{1}{1-x^{\prime}}\left(1, x^{\prime}, \vec{x}^{\prime \prime}\right)\right]=[1, x] \rightarrow \frac{\vec{x}^{\prime \prime}}{1-x^{\prime}} .
$$


This amounts to taking a lift of $x$ in the lightcone, denoted as $\tilde{x}$, such that $\langle\tilde{x}, \wp\rangle=-1$, and then projecting $\tilde{x}$ to the orthogonal complement of $\{(1,1, \overrightarrow{0}),(1,-1, \overrightarrow{0})\}$.

Based on this observation, we need only to take two arbitrary points $p=[\wp], p *=$ $[\wp *] \in \mathbb{S}^{m+2}$, which can always be expressed as

$$
\wp=(1,1, \overrightarrow{0}), \wp^{*}=(1,-1, \overrightarrow{0}),\left\langle\wp, \wp^{*}\right\rangle=-2,
$$

with respect to a suitable Lorentz coordinate system. The desired local lift $\tilde{x}$ of $O_{\xi}$ in (42) is given by

$$
\begin{gathered}
\tilde{x}=\frac{1}{\sigma} O_{\xi}=\frac{1}{\sigma}(\wp-\langle\wp, \xi\rangle \bar{\xi}-\langle\wp, \bar{\xi}\rangle \xi), \\
\sigma \triangleq-\left\langle O_{\xi}, \wp\right\rangle=-2\langle\wp, \xi\rangle\langle\wp, \bar{\xi}\rangle .
\end{gathered}
$$

And the explicit mapping to $\mathbb{R}^{m+2}$ is

$$
\begin{aligned}
\tilde{X} & =\tilde{x}+\frac{1}{2}\langle\tilde{x}, \wp\rangle \wp^{*}+\frac{1}{2}\left\langle\tilde{x}, \wp^{*}\right\rangle \wp \\
& =-\frac{1}{2} \wp^{*}-\frac{1}{\langle\wp, \xi\rangle} \xi-\frac{1}{\langle\wp, \bar{\xi}\rangle} \bar{\xi}-\frac{\left\langle\wp^{*}, \xi\right\rangle}{4\langle\wp, \xi\rangle} \wp-\frac{\left\langle\wp^{*}, \bar{\xi}\right\rangle}{4\langle\wp, \bar{\xi}\rangle} \wp \\
& =\frac{1}{2}(X+\bar{X})
\end{aligned}
$$

This is exactly the real part of

$$
X=\frac{-1}{2\langle\xi, \wp\rangle}\left(2 \xi+\langle\xi, \wp\rangle \wp^{*}+\left\langle\xi, \wp^{*}\right\rangle \wp\right)
$$

This $X$ depends on the conformal Gauss map [ $\xi]$, which is a mapping from a Riemann surface $\bar{M}^{2}$ to $Q_{+}^{m+2} \subset \mathbb{C} P^{m+3}$ by Theorem 5.1. So one may regard this is a mapping $X: \bar{M}^{2} \rightarrow$ $\mathbb{C}_{1}^{m+4}$. We have the following conclusions:

First, this is indeed a mapping to $\mathbb{C}^{m+2}$ because $\langle X, \wp\rangle=\left\langle X, \wp^{*}\right\rangle=0$.

Second, this complex vector-valued function is holomorphic. This follows from Theorem 5.1 that $[\xi]$ is holomorphic, i.e., $\xi_{\bar{z}}=\lambda \xi$ for local coordinate $z$ and local function $\lambda$. Together with (43), it implies that $X_{\bar{z}}=0$.

Thirdly, $X_{z}$ is isotropic. We need only to re-write (43) as

$$
X=-\frac{1}{2} \wp^{*}-\tilde{\xi}-\frac{1}{2}\left\langle\tilde{\xi}, \wp^{*}\right\rangle \wp, \tilde{\xi} \triangleq \frac{1}{\langle\xi, \wp\rangle} \xi
$$

Since $\langle\tilde{\xi}, \wp\rangle=1$ is constant, $\left\langle\tilde{\xi}_{z}, \wp\right\rangle=0$. Moreover, for this codimension two Wintgen ideal submanifold, Theorem 5.1 already tells us $\xi, \xi_{z}$ are isotropic. So $\tilde{\xi}_{z}$ is isotropic. As the consequence, $X_{z}=\tilde{\xi}_{z}-(\cdots) \wp$ is isotropic. We also know $\left|X_{z}\right|^{2}>0$ because $\left|\xi_{z}\right|^{2}>0$ by Theorem 5.1.

From these three conclusions we know $X: \bar{M}^{2} \rightarrow \mathbb{C}^{m+2}$ is an isotropic, holomorphic vector-valued function. So its real part $\tilde{X}$ defines an immersed minimal surface in $\mathbb{R}^{m+2}$.

In [7] the inverse procedure is also given, namely the construction of Wintgen ideal submanifolds of codimension two from Euclidean minimal surfaces. 
Instead of repeating their description at here, we will give an interpretation of this relationship between these two classes of geometric objects. By our main results in the previous section, the first class (Wintgen ideal $M^{m} \rightarrow \mathbb{S}^{m+2}$ ) is essentially the same as holomorphic 1 -isotropic curves in $Q_{+}^{m+2}$. On the other hand, the second class (minimal $\bar{M}^{2} \rightarrow \mathbb{R}^{m+2}$ ) is well-known to be identical with holomorphic 1-isotropic curves in $\mathbb{C}^{m+2}$. Thus it suffices to establish a correspondence between these two classes of holomorphic 1-isotropic curves.

The proof of Theorem 6.1 already included an explicit correspondence between $Q^{m+2}$ and $\mathbb{C}^{m+2} \subset \mathbb{C}_{1}^{m+4}$ as below:

$$
\pi:[\xi] \mapsto X=\frac{-1}{2\langle\xi, \wp\rangle}\left(\langle\xi, \wp\rangle \wp^{*}+\left\langle\xi, \wp^{*}\right\rangle \wp+2 \xi\right),
$$

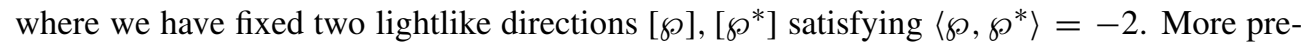
cisely, the domain of $\pi$ is an open dense subset of $Q^{m+2}$ where $\langle\xi, \wp\rangle \neq 0$; the image is the orthogonal complement of $\left\{\wp, \wp^{*}\right\}$.

To find the inverse of $\pi$, put $\wp, \wp^{*}$ as before and $X=\left(0,0, X_{1}, \ldots, X_{m}\right) \in \mathbb{C}^{m+2} \subset$ $\mathbb{C}_{1}^{m+4}$. The inverse $\pi^{-1}$ is then given by

$$
\pi^{-1}: X \mapsto[\xi], \quad \xi=\wp^{*}+\langle X, X\rangle \wp+2 X .
$$

It is easy to verify that the $\xi=\xi_{1}-i \xi_{2}$ given above satisfies

$$
\langle\xi, \xi\rangle=0,\langle\xi, \bar{\xi}\rangle=4\langle X, \bar{X}\rangle-2\langle\xi, \xi\rangle-2\langle\bar{\xi}, \bar{\xi}\rangle=4\left|\xi_{2}\right|^{2} .
$$

So $[\xi]$ defined above is in $Q^{m+2}$ as desired. By the assumption $\langle X, \wp\rangle=\left\langle X, \wp^{*}\right\rangle=$ $0,\left\langle\wp, \wp^{*}\right\rangle=-2$, it is straightforward to show that (45) and (44) are inverse mappings to each other.

Indeed (44) is a complex version of the classical stereographic projection. Take $\wp=$ $(1,1, \overrightarrow{0}), \wp^{*}=(1,-1, \overrightarrow{0})$ and the lift $\xi=\left(1, \xi^{\prime}, \vec{\xi}^{\prime \prime}\right)$. Then (44) and (45) read as

$$
\begin{gathered}
\pi:\left(1, \xi^{\prime}, \vec{\xi}^{\prime \prime}\right) \mapsto\left(0,0, \frac{\vec{\xi}^{\prime \prime}}{1-\xi^{\prime}}\right), \\
\pi^{-1}:(0,0, X) \mapsto\left(|X|^{2}+1,|X|^{2}-1,2 X\right) .
\end{gathered}
$$

These formulas are similar to the classical stereographic projection. In particular, when $X \in$ $\mathbb{R}^{m+2}$ we get the old version between the projective lightcone and the Euclidean space.

THEOREM 6.3. Fix $\wp=(1,1, \overrightarrow{0}), \wp^{*}=(1,-1, \overrightarrow{0}) \in \mathbb{R}_{1}^{m+4}$. Then the complex stereographic projection in (44) and its inverse (45) establish a correspondence between holomorphic 1-isotropic curves in $Q_{+}^{m+2} \subset \mathbb{C} P_{1}^{m+3}$ and holomorphic 1-isotropic curves in $\mathbb{C}^{m+2}$. This is a one-to-one correspondence up to the choice of the poles $\wp, \wp *$ *

PROOF. Let [ $\xi]: \bar{M}^{2} \rightarrow Q_{+}^{m+2}$ be a holomorphic 1-isotropic curve. The conclusion that $\pi[\xi]=X$ is holomorphic and 1-isotropic is proved almost the same as that of Theorem 6.1. Conversely, given a vector-valued function

$$
X=\left(0,0, X_{1}, \ldots,\right): \bar{M}^{2} \rightarrow \mathbb{C}^{m+2} \subset \mathbb{C}_{1}^{m+4},
$$


which is holomorphic and 1-isotropic, i.e., $X_{\bar{z}}=0,\left\langle X_{z}, X_{z}\right\rangle=0$. Then $\xi=\wp^{*}+\langle X, X\rangle \wp+$ $2 X$ defined by (45) obviously satisfy $\xi_{\bar{z}}=0,\langle\xi, \xi\rangle=0$. From $\xi_{z}=2\left\langle X_{z}, X\right\rangle_{\wp}+2 X_{z}$ it is 1 -isotropic. This finishes the proof.

REMARK 6.4. By Theorem 6.1 and Theorem 6.3, Wintgen ideal submanifolds of codimension two are constructed from two equivalent geometric objects, i.e., holomorphic 1isotropic curves in $Q_{+}^{m+2}$ or in $\mathbb{C}^{m+2}$. The first description is given by us. It has the advantage of being invariant under the Möbius transformations. Combined with Thereom 5.1 and Theorem 5.2, it captures the global structure of a Wintgen ideal submanifold of codimension two. From another point of view [7], minimal surfaces in $\mathbb{R}^{m+2}$ and holomorphic curves in $\mathbb{C}^{m+2}$ are easy to describe explicitly, which would enable us to construct examples of Wintgen ideal submanifolds efficiently.

\section{REFERENCES}

[ 1 ] R. BRYANT, A duality theorem for Willmore surfaces, J. Differential Geom. 20 (1984), 20-53.

[2] R. BRYAnt, Surfaces in Conformal Geometry, The mathematical heritage of Hermann Weyl (Durham, NC, 1987), 227-240, Proc. Sympos. Pure Math. 48, Amer. Math. Soc., Providence, RI, 1988.

[ 3 ] R. BRYANT, Some remarks on the geometry of austere manifolds, Bol. Soc. Brasil. Mat. (N.S.) 21 (1991), 133-157.

[ 4 ] B. CHEN, Classification of Wintgen ideal surfaces in Euclidean 4-space with equal Gauss and normal curvatures, Ann. Global Anal. Geom. 38 (2010), 145-160.

[ 5 ] M. DAjCzer ANd R. Tojeiro, A class of austere submanifolds, Illinois J. Math. 45 (2001), no. 3, 735-755.

[6] M. Dajczer And R. Tojeiro, All superconformal surfaces in $R^{4}$ in terms of minimal surfaces, Math. Z. 261 (2009), no. 4, 869-890.

[ 7 ] M. DAJCZER AND R. TojeIro, Submanifolds of codimension two attaining equality in an extrinsic inequality, Math. Proc. Cambridge Philos. Soc. 146 (2009), no. 2, 461-474.

[ 8 ] P. J. De smet, F. Dillen, L. Verstraelen and L. Vrancken, A pointwise inequality in submanifold theory, Arch. Math. 35 (1999), 115-128.

[9] F. Dillen, J. Fastenakels and J. VAn Der Veken, Remarks on an inequality involving the normal scalar curvature, Pure and applied differential geometry PADGE 2007, 83-92, Ber. Math., Shaker Verlag, Aachen, 2007.

[10] J. GE AND Z. TANG, A proof of the DDVV conjecture and its equality case, Pacific J. Math. 237 (2008), 87-95.

[11] I. Guadalupe And L.RodríGuez, Normal curvature of surfaces in space forms, Pacific J. Math. 106 (1983), 95-103.

[12] T. LI, X. MA AND C. Wang, Wintgen ideal submanifolds with a low-dimensional integrable distribution (I), Front. Math. China 10 (2015), no. 1, 111-136.

[13] T. Choi AND Z. LU, On the DDVV conjecture and the comass in calibrated geometry (I), Math. Z. 260 (2008), 409-429.

[14] Z. LU, Normal Scalar Curvature Conjecture and its applications, J. Funct. Anal. 261 (2011), 1284-1308.

[15] X. MA AND Z. XIE, The Moebius geometry of Wintgen ideal submanifolds, Proceedings of the ICM 2014 Satellite Conference on Real and Complex Submanifolds (Daejeon, 2014), 411-425, Springer, 2014.

[16] M. Petrovié-torgašev and L. Verstraelen, On Deszcz symmetries of Wintgen ideal Submanifolds, Arch. Math. 44 (2008), 57-67.

[17] B. RouXEL, Harmonic spheres of a submanifold in Euclidean space, Proceedings of the 3rd Congress of Geometry (Thessaloniki, 1991), 357-364, Aristotle Univ. Thessaloniki, 1992. 
[18] C. WANG, Möbius geometry of submanifolds in $S^{n}$, Manuscripta Math. 96 (1998), 517-534.

[19] P. Wintgen, Sur l'inégalité de Chen-Willmore, C. R. Acad. Sci. Paris Sér. A-B 288 (1979), 993-995.

[20] Z. XIE, T. LI, X. MA AND C. WANG, Möbius geometry of three-dimensional Wintgen ideal submanifolds in $\mathbb{S}^{5}$, Sci. China Math. 57 (2014), 1203-1220.

DEPARTMENT OF MATHEMATICS

BEIJING INSTITUTE OF TECHNOLOGY

BEIJING 100081

People's Republic of China

E-mail address: litz@bit.edu.cn

School of Mathematics AND Computer SCIENCE \& FJKLMAA

FUJIAN NORMAL UNIVERSITY

FUZHOU 350108

PEOPLE's Republic OF CHINA

E-mail address: cpwang@fjnu.edu.cn
School of Mathematical Sciences

PEKING UNIVERSITY

BEIJING 100871

PEOPLE's REPUBLIC OF CHINA

E-mail address: maxiang@math.pku.edu.cn

Department of Mathematics

China University OF Mining AND

TECHNOLOGY (BEIJING)

BEIJING 100083

People's Republic of ChinA

E-mail address: xiezhenxiao@126.com 